Article

\title{
Quantitative Association between Nighttime Lights and Geo-Tagged Human Activity Dynamics during Typhoon Mangkhut
}

\author{
Zhang Liu ${ }^{1,2}$, Yunyan Du ${ }^{1,2, *}$, Jiawei $\mathrm{Yi}^{1,2}{ }^{\text {, Fuyuan Liang }}{ }^{3}$, Ting Ma ${ }^{1,2}$ and Tao Pei ${ }^{1,2} \mathbb{C}$ \\ 1 State Key Laboratory of Resources and Environmental Information System, Institute of Geographical \\ Sciences and Natural Resources Research, Chinese Academy of Sciences, Beijing 100101, China \\ 2 College of Resources and Environment, University of Chinese Academy of Sciences, Beijing 100049, China \\ 3 Department of Earth, Atmospheric, and Geographic Information Sciences, Western Illinois University, \\ Macomb, IL 61455, USA \\ * Correspondence: duyy@Ireis.ac.cn
}

Received: 17 July 2019; Accepted: 4 September 2019; Published: 6 September 2019

\begin{abstract}
The daily nighttime lights (NTL) and the amount of location-service requests (NLR) data have been widely used as a proxy for measures of disaster-induced power outages and geo-tagged human activity dynamics. However, the association between the two datasets is not well understood. In this study, we investigated how the NTL signals and geo-tagged human activities changed in response to Typhoon Mangkhut. The confusion matrix is constructed to quantify the changes of the NLR in response to Typhoon Mangkhut, as well as the changes of the NTL signals at the grid level. Geographically-weighted regression and quantile regression were used to examine the associations between the changes of the NTL and the NLR at both grid and county levels. The quantile regressions were also used to quantify the relationships between the dimmed NTL signals and the change of the NLR in disaster damage estimates at the county level. Results show that the percent of the grids with anomalous human activities is significantly correlated with the nearby air pressure and wind speed. Geo-tagged human activities varied in response to the evolution of Mangkhut with significant areal differentiation. Over $69.3 \%$ of the grids with significant human activity change is also characterized by declined NTL brightness, which is closely associated with abnormal human activities. Significant log-linear and moderate positive correlations were found between the changes of the NTL and NLR at both the grid and county levels, as well as between the county-level changes of NLR/NTL and the damage estimates. This study shows the geo-tagged human activities are closely associated with the changes of the daily NTL signals in response to Typhoon Mangkhut. The two datasets are complimentary in sensing the typhoon-induced losses and damages.
\end{abstract}

Keywords: NPP-VIIRS DNB; social media; natural disaster; typhoon; damage assessment

\section{Introduction}

Rapid damage assessment is crucial for emergency and disaster response. Over the past 20 years, satellite images have been widely used for near real-time monitoring and rapid assessing disasters-induced damages and losses [1]. Optical and radar remote sensing data are usually used to assess physical damages such as ground deformation or structural destruction [2-5]. By contrast, nighttime lights remote sensing data, which record artificial nocturnal radiances across human settlements [6], could be used to infer human activity disturbance. Changes in nighttime lights resulting from disaster-induced infrastructure damages [7-9], power outages [10-12], and emergency evacuation $[13,14]$ could be derived from short-term variations in the National Polar-orbiting Partnership Visible Infrared Imaging Radiometer Suite (NPP-VIIRS) day/night band (DNB) data, which 
in turn can be used to infer disaster-induced losses and damages. The well-calibrated DNB daily data could be used to delineate the spatial extent and identify the recovery trend of a hurricane-induced power outage, and the results are well consistent with the power company survey data [10,12]. The DNB daily data are found to be useful for detecting damages caused by earthquakes, storms, and floods [13]. However, due to the constraints of spectral, spatial, and temporal resolutions of the sensors, satellite-derived artificial lighting data are not fully exploited in the context of emergency response [15]. Furthermore, the usefulness could be obstructed by high cloud coverage during and after the hazards [13], particularly for hurricanes and floods.

Recently booming social media data, when integrated with remote sensing images, have shown their potential in studying emergency response. Human activities could be inferred from the location-aware big data, which are typically derived from mobile phone records, smart card usage and social media uses [16,17]. More importantly, location-aware big data provide almost real-time records of public responses to a natural disaster in both the virtual and physical worlds [18]. Such advantages make the location-aware big data a perfect proxy for measures of human's collective response to natural disasters [19]. Previous studies have shown the quantitative relationships between location-aware big data and natural disasters themselves and the damages they caused. For example, disaster-related social media data are significantly correlated with the actual rainfall of a rainstorm, the shaking intensity of an earthquake, and the atmospheric pressure of a hurricane [20-24]. Strong positive relationships have also been found between the proximity to the hurricane's path and hurricane-related twitter activity, and between per-capita twitter activity and per-capita economic damage inflicted by the hurricane $[18,25]$.

Many previous studies have integrated social media data and remote sensing imagery to monitor disasters and assess disaster-induced damages. The VIIRS NTL data and geo-tagged tweets were integrated to identify the streets affected by power outages during Hurricane Sandy, which is impossible if only the NTL data were used [26]. Social media data were also combined with remote sensing imagery to improve transportation damage assessment during the 2013 Colorado floods [27,28] and to estimate and forecast rainfall and runoff [29,30]. A transfer learning framework was proposed for urban waterlogging analysis using social media and satellite data [31]. A Bayesian statistical model was developed to estimate the probability of flood inundation using social media data, optical remote sensing and high-resolution terrain data [32]. Multi-source satellite and Twitter data were combined to delineate the flood extent on a daily basis [33]. Similar studies can also be found in [34-38]. These studies showed that social media data is valuable in providing disaster-related information at locations that are not being sensed by satellite sensors. Furthermore, integration of remote sensing and social media data can significantly shorten emergency response time. However, it is not clear how remote sensing data (e.g., daily NTL signals) and human activities are quantitatively correlated at multiple scales during and after a natural disaster.

A natural disaster may cause a power outage, which could be seen from the variations in the daily NTL signals. The power outage would also significantly disturb geo-tagged human activities, which could be inferred from the variations in the location-aware data. Examining the association between the variations in the daily NTL signals and location-aware data would help us better understand and assess the impacts of a natural disaster on human beings from multiple perspectives. The association would also help us determine whether the location-aware data could be used as a proxy of NTL data, which could be missing due to the inclement weather accompanied with the disasters [13].

The primary objective of this study is to investigate the association between the NTL data and the collective geo-tagged human activities in response to Typhoon Mangkhut at grid and county levels. A procedure was proposed to effectively detect the abrupt changes in the hourly NLR time series at the grid level. We also examined the relationships between anomaly geo-tagged human activities and the typhoon wind speed and air pressure. Moreover, geographically weighted regression (GWR) and quantile regression were used to examine the relationships between the decline in NTL (dNTL) and the change of the number of location request (cNLR) at both grid and county levels. A partitioning 
algorithm combining the Jenks natural breaks optimization and bivariate choropleths was used to characterize the spatial differentiation between the variations in the NTL signals and geo-tagged human activities. Finally, we used the quantile regression to quantify the relationships between the NTL and NLR signals and the damage estimates at the county level.

\section{Materials and Methods}

\subsection{Geographical Context and Typhoon Mangkhut}

This study examined the impacts of Typhoon Mangkhut on three provinces (Guangdong, Guangxi, and Hainan) and two special administrative regions (Macao and Hong Kong) in Southern China. Our study area includes one of the most rapidly urbanized and developed regions in China, the Pearl River Delta Metropolitan Region (PRDMR), which covers an area of 54,574 $\mathrm{km}^{2}$ and includes Hong Kong, Macao, and nine cities in Guangdong Province (Guangzhou, Shenzhen, Foshan, Dongguan, Huizhou, Zhuhai, Zhongshan, Zhaoqing, and Jiangmen). The residential population of PRDMR reached 68 million as of 2016. The GDP of PRDMR in 2016 is 145.024 billion US\$, accounting for 70\% and $12 \%$ of the total GDP of Guangdong Province and China, respectively [39].

Typhoon Mangkhut was a strong tropical cyclone when it first hit Southern China (Figure 1). It made landfall in Jiangmen, Guangdong, at 5:00 pm on 16 September 2018 with a maximum wind speed of $162 \mathrm{~km} / \mathrm{h}$. Mangkhut lasted as a typhoon for the first six hours after the landfall. It then rapidly weakened to a tropical storm while moving westward and finally dissipated in Guangxi on 17 September. It was the strongest typhoon to strike Hong Kong since Typhoon Ellen in 1983. It triggered the issue of a No.10 (the highest level) warning signal for $10 \mathrm{~h}$. Typhoon Mangkhut caused about 21,000 homes to lose electrical power and 7000 homes to lose internet service in Macau. On mainland China, Mangkhut killed six people and caused over 2.45 million people to evacuate and more than US $\$ 1.99$ billion worth of damage [40].

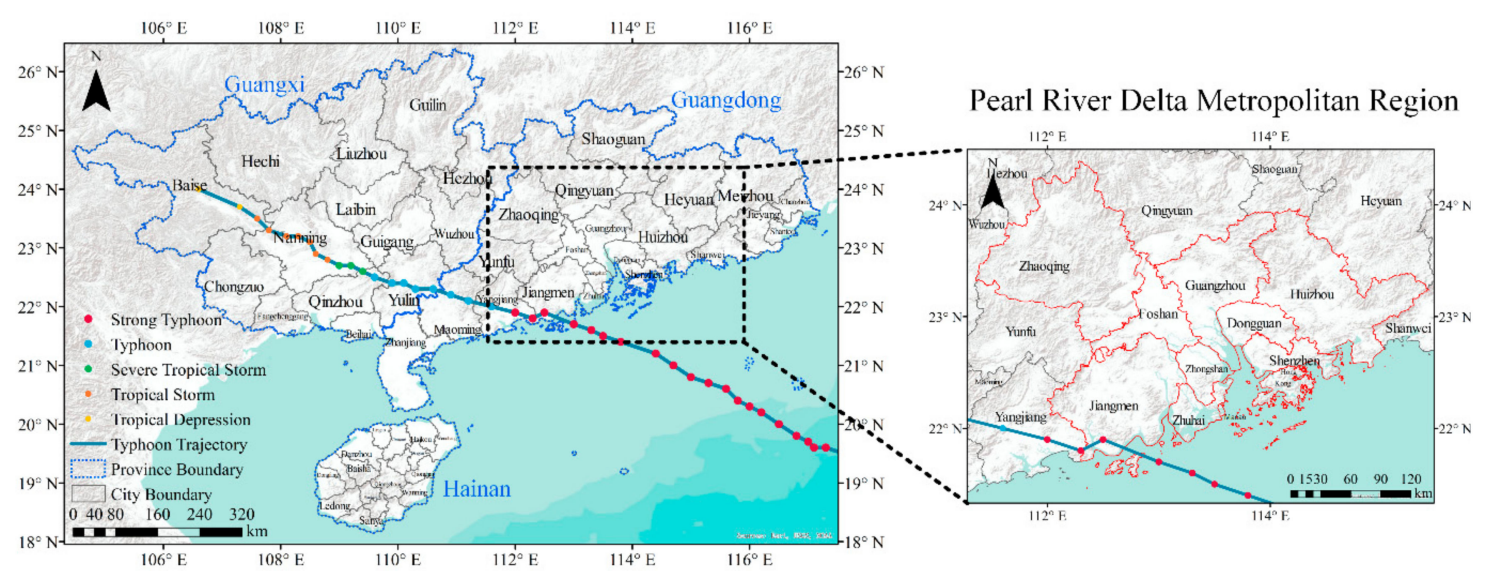

Figure 1. The track of Typhoon Mangkhut over the area affected. The track was obtained from the National Meteorological Center of China Meteorological Administration [41].

\subsection{Data}

\subsubsection{The NPP-VIIRS DNB Daily Data}

We used two NPP-VIIRS datasets in this study: the DNB Sensor Data Record (SDR) and the Cloud Cover Layer (CCL) Environmental Data Record (EDR), which were retrieved from the National Oceanic and Atmospheric Administration (NOAA)'s Comprehensive Large Array-data Stewardship System [42]. The DNB SDR records the gridded radiance with a spatial resolution of $\sim 750 \mathrm{~m}$ across the entire swath, which can be used to quantify radiance emitted from multiple light sources. The CCL data, in particular, the summed cloud cover (SCC), was used to filter out the cloud-contaminated DNB data. We also obtained the Moderate Resolution Imaging Spectroradiometer (MODIS) Terre 
V005 500 m surface reflectance product (MCD09A1) from the NASA Earthdata website [43]. The MODIS MCD09A1 was used as auxiliary data to calculate the reflected lunar radiance recorded by the DNB sensor.

The NTL signals were derived from DNB data. We calibrated the raw DNB data using the method proposed in [13] by first eliminating contamination of cloud coverage and then lunar radiance. There is no evidence showing the DNB images of our study area were contaminated by lights from fishing boats, gas flares, lighting, auroras, and volcanic and stray light within the time period of interest in this study. However, cloud contamination does exist. In this study, we excluded the grids with an SCC value over 0.01 . We then computed the lunar radiance from the initial DNB radiance using the method proposed in [10]. The method computes the lunar radiance recorded by the DNB sensors with the assistance of a top-of-atmosphere (TOA) spectral lunar irradiance model (MT2009) that was developed in [44]. The MODIS MCD09A1 was then used to replace the TOA reflectance and quantify the band averaged surface reflectance. We then visually assessed all images and discarded those with other issues such as being affected by heavy aerosol. Finally, the DNB daily images acquired one month prior to and 10 days after Typhoon Mangkhut (Table 1) were averaged to create pre- and post-disaster composite datasets, respectively. The NPP-VIIRS DNB daily data calibrated by the above-mentioned image pre-processing procedure have proved to be useful in detecting disaster-induced infrastructure damages and power outages [13].

Table 1. The dates of the pre- and post-typhoon DNB images that were used in this study.

\begin{tabular}{ccccccccc}
\hline \multicolumn{1}{c}{ Pre-Typhoon } & \multicolumn{3}{c}{ Post-Typhoon } \\
\hline Date & 3 Sept. & 4 Sept. & 10 Sept. & 13 Sept. & 14 Sept. & 15 Sept. & 18 Sept. & 19 Sept. \\
\hline $\begin{array}{c}\text { Days before and } \\
\text { after typhoon }\end{array}$ & -13 & -12 & -6 & -3 & -2 & -1 & +2 & +3 \\
\hline
\end{tabular}

\subsubsection{The Tencent's Location-Aware data}

We collected one-month location-aware data (1 to 30 September 2018) from Tencent's location data portal [45]. Tencent is the most popular location-based social network service provider in China and has over 0.7 billion active users. The gridded dataset records the number of location-service requests (NLR) initiated by social media users for a variety of purposes, such as navigation, social media check-ins, taxi hailing, and uploading a geo-tagged microblog and/or a picture. The dataset has a spatial resolution of $\sim 1 \mathrm{~km} \times 1 \mathrm{~km}$ and an hourly temporal resolution. Recent studies have shown that the changes in the daily NLR can explain more than $80 \%$ of the variation in the number of tourists in 11 tourist attractions over a $~ 60$-day time period [46-48]. In this study, we used the NLR data as a proxy of the collective geo-tagged human activities (hereafter referred to as human activities unless expressly stated otherwise) at a short-time scale.

\subsubsection{Other Ancillary Data}

We collected statistical data of the damages and losses, including the direct economic losses, the population affected, and emergency resettlement population from the local government websites of the 147 counties that were affected by Typhoon Mangkhut. The wind speed probability product, which was obtained from the National Hurricane Center [49], is used to determine the geographic extent that was impacted by the typhoon wind. This product estimates the probability that any given location would experience 34-, 50-, or 64-knot winds (i.e., tropical-storm-force winds, storm-force winds, or hurricane-force winds) over a given forecast time period [50,51]. The wind speed and air pressure of the weather stations within our study area were obtained from NOAA [52].

We also obtained the ambient (average over 24 h) 2016 and 2017 population from the LandScan global database developed by the Oak Ridge National Laboratory. The LandScan population dataset has a spatial resolution of $\sim 1 \mathrm{~km} \times 1 \mathrm{~km}$. The Climate Change Initiative Land Cover $2015 \mathrm{Map}$, which 
has a spatial resolution of $\sim 300 \mathrm{~m}$ and was developed by the European Space Agency, was used to exclude water grids from this study.

\subsection{Methods}

We followed the methodology framework in Figure 2 to first identify the grids with a stable NLR, from which the abrupt changes in the gridded hourly time series were identified. Next, we quantified the spatial patterns of the human activities in response to the typhoon and then evaluated the correlations between the NLR and NTL signals at the grid and county levels. Lastly, we assessed the association between the NLR and NTL signals with the typhoon-induced losses and damages at the county level.

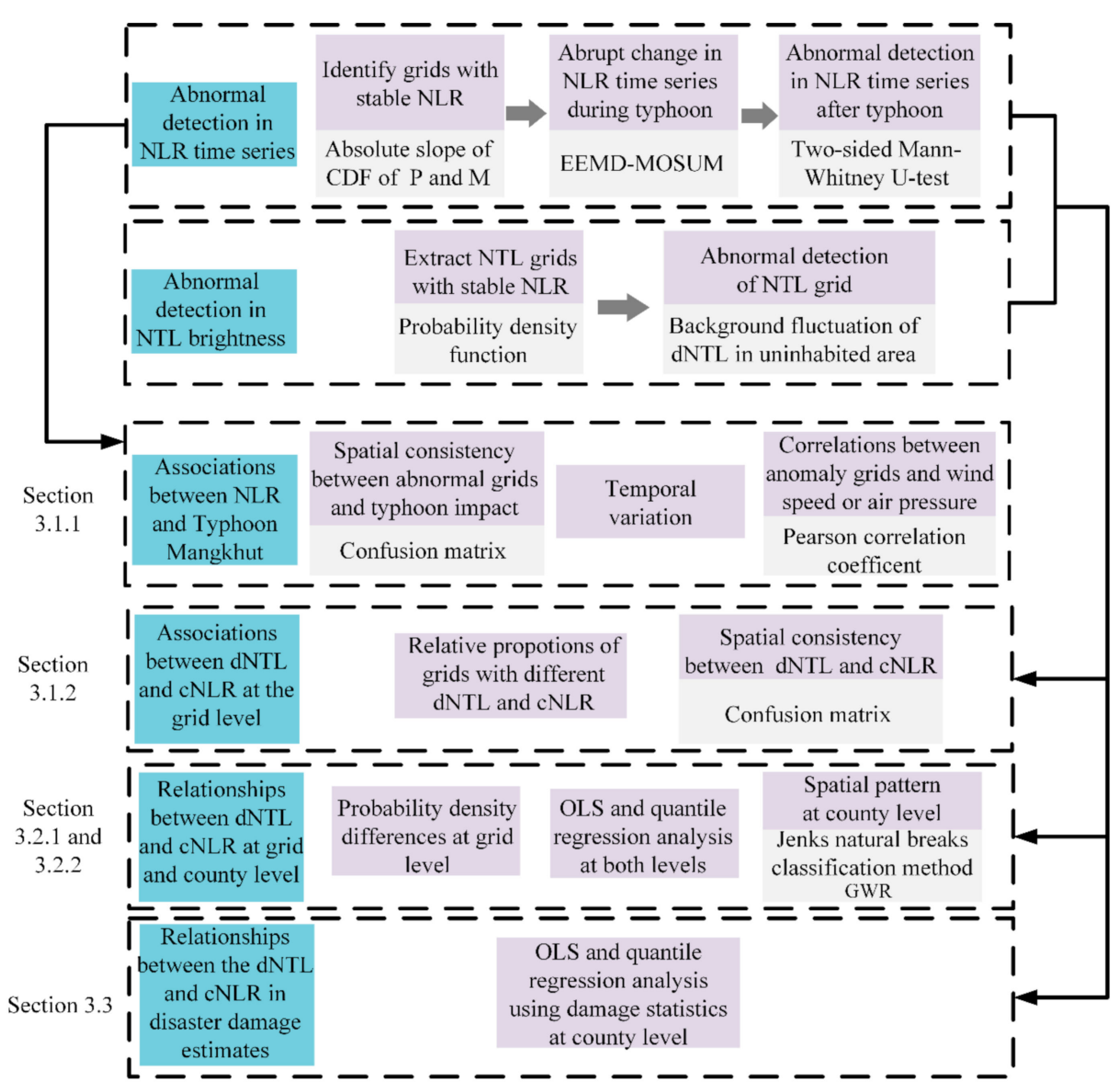

Figure 2. A flow chart showing the data analysis process in this study.

\subsubsection{Detect Abrupt Changes in the Hourly Time Series of the Stable Grids}

We first identified all grids with a stable NLR (hereafter these grids are referred as to stable grids unless expressly stated otherwise) using the method we proposed in [48]. In total, we identified 83,230 stable grids in this study (Figure S1), accounting for $20.6 \%$ of the total number of grids $(403,800)$ over our study area.

We developed a procedure Ensemble Empirical Mode Decomposition-Moving Sum (EEMD-MOSUM) and used it to detect the abrupt changes in the hourly NLR time series of the stable grids at the grid level (Figure 3). The procedure first decomposes the time series into a series of orthogonal components [53,54], also known as the instinct mode functions (IMFs), and a residue (Figure S2). For the time series with a length of $N$, a complex time series could be decomposed to less than $\log _{2} N$ IMFs. Each IMF represents an oscillatory mode of the time series, and the residue stands for the overall trend. IMF1 has the highest frequency and usually contains rapid abnormal changes and 
noise. Other IMFs, if chosen appropriately, can distinguish the seasonal from the trend components in the time series. The seasonal component varies at or near the seasonal frequency (i.e., $1 \mathrm{~Hz}$, one cycle per day), whereas the trend component is the low-frequency variation along with the non-stationary long-term changes.

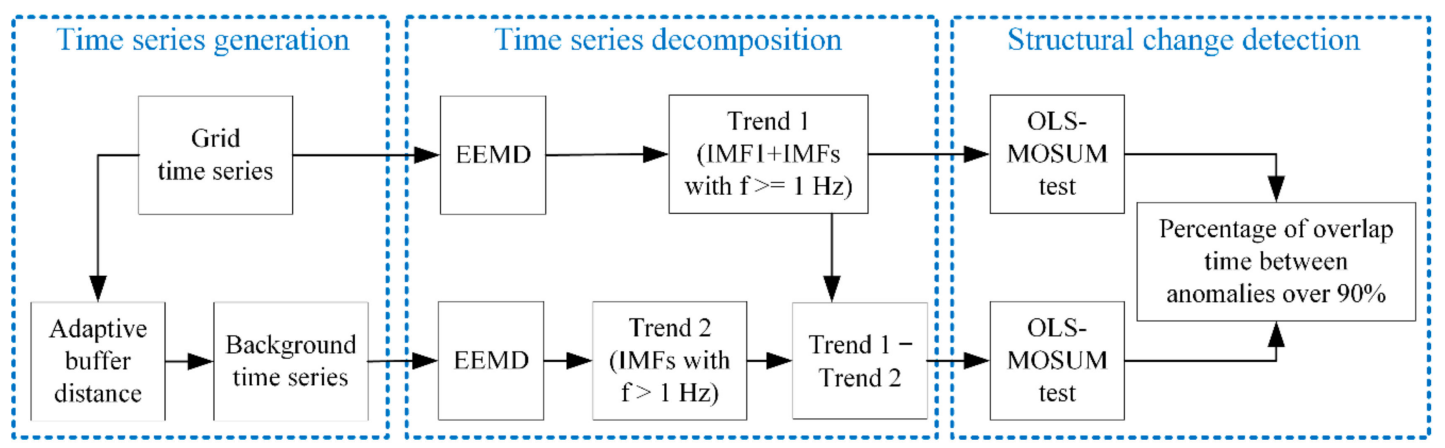

Figure 3. The framework of the EEMD-MOSUM data processing procedure.

We first decomposed the NLR time series of the stable grids (Figure 4a) into eight IMFs and a residue. The IMFs were separately used to generate the trend $T_{1}$ (IMF 4-8 and the residue, frequency $\leq 1 \mathrm{~Hz}$ ), seasonal $S_{1}\left(\mathrm{IMF} 2-3\right.$, frequency $>1 \mathrm{~Hz}$ ), and the remainder $e_{1}$ (IMF 1 ) components. The high-frequency IMF 4 (frequency $=1 \mathrm{~Hz}$ ) was added to the trend component to improve the accuracy of abrupt change detection. The ordinary least squares residuals-based moving sum (OLS-MOSUM) test is then used to detect the abrupt changes in the component $T_{1}+e_{1}$ (Figure $4 \mathrm{c}$ ). The component $T_{1}+e_{1}$ remains stable if the MOSUM process randomly fluctuates around zero and $p \geq 0.05$. When the MOSUM process significantly deviates from $0(p<0.05)$, a structural change occurs and the corresponding time series segments would be retained as abrupt change candidates.
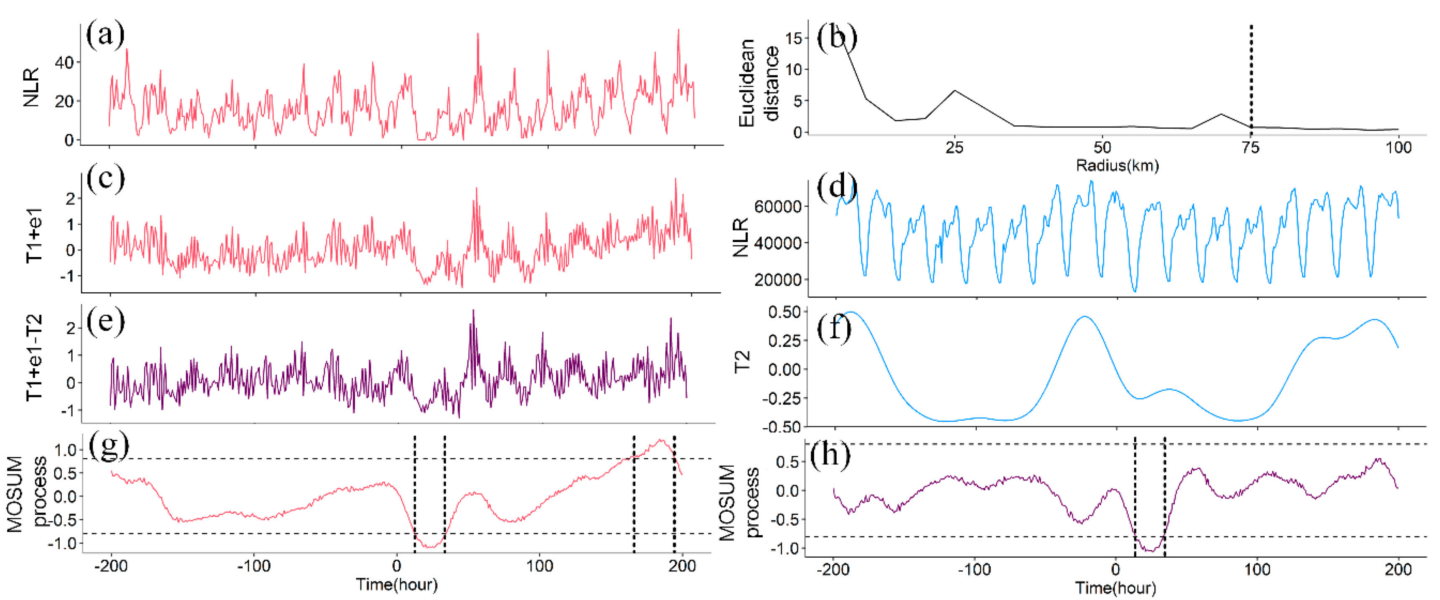

Figure 4. Detection of the abrupt changes in the NLR time series using the EEMD-MOSUM procedure. (a) The original NLR time series. (b) The similarity (measured by the Euclidean distance) between two adjacent time series that were constructed within different-radius buffer zones. (c) Component $T_{1}+e_{1}$ of the original NLR time series. (d) The NLR time series within the 70-km buffer zone. (e) Component $T_{1}+e_{1}-T_{2}$. (f) Component $T_{2}$ the NLR time series within the 70-km buffer zone. (g) Abrupt changes in component $T_{1}+e_{1}$ detected by the OLS-MOSUM test. (h) Abrupt changes in component $T_{1}+e_{1}-T_{2}$ detected by the OLS-MOSUM test.

Tencent's gridded NLR time series are stained with certain systemic fluctuations, which affect the stability of the dataset and then the accuracy of abrupt change detection. In this study, we used the trend of the NLR time series within a grid's buffer zone to reduce the impact of the systematic 
fluctuations. When the buffer distance of a specific grid increases, the two spatially adjacent gridded time series become more similar (i.e., the Euclidean distance decreases) and the time series become more stable once the distance is over a threshold. In this study, we used a distance threshold of $\sim 70 \mathrm{~km}$, which is close to the average of the radii of the cities in our study area.

We then decomposed the NLR time series of every stable grid within its buffer zone (Figure $4 \mathrm{~d}$ ) into eight IMFs and one residue. The IMFs 5-8 and the residue (frequency $<1 \mathrm{~Hz}$ ) were used to generate the trend $T_{2}$ (Figure $4 \mathrm{f}$ ), which was used to eliminate the systematic fluctuations in the component $T_{1}+e_{1}$. The OLS-MOSUM test was again applied to detect the abrupt changes in the component $T_{1}+e_{1}-T_{2}$. If the grid's abrupt changes are significant in relation to the systematic fluctuations, the OLS-MOSUM test results of the component $T_{1}+e_{1}$ should be similar to those of $T_{1}+e_{1}-T_{2}$. The timing of the abrupt changes detected from the components $T_{1}+e_{1}$ and $T_{1}+e_{1}-T_{2}$ should be very close yet with certain offsets. Our experiments showed that an anomaly would be a true one if its timing offset against its neighbor is less than $90 \%$ of its duration.

\subsubsection{Identify the Spatial Patterns of the Human Activities in Response to Typhoon Mangkhut}

We used $80 \%$ probability of the storm-force wind (50-knot wind speed) to define the typhoon impacting zone. A confusion matrix was constructed to evaluate whether the grids within the impacting zone show more significant NLR abrupt changes than those beyond.

We also examined the relationships between the percent of the anomaly grids and the wind speed/air pressure around select weather stations. The grids were categorized into different groups depending on the time ranges and whether they are located within a specifically-defined region [22,23]. In this study, the region is defined as a $70 \mathrm{~km}$-buffer zone around each weather station. The $70 \mathrm{~km}$ represents the average geographical radius of all cities within our study area. The time range is specified as $24 \mathrm{~h}$ before and after the typhoon made landfall. We normalized the hourly anomalies of each buffer zone to hourly average values using a six-hour moving time window [22] to eliminate the effect of spatiotemporal sparsity of the anomaly grids. In the end, the time series anomalies, wind speed, and air pressure are all transformed to hourly granularity for each station. The Pearson correlation coefficients were then calculated to measure the correlations between the anomalies and the wind speed and air pressure, respectively.

\subsubsection{Quantify the Relationships Between the NLR and NTL Signals at the Grid and County Levels}

Two indices, the decline in the NTL (dNTL) and the change of the NLR (cNLR), were used to quantify the differences between the pre- and post-typhoon NTL brightness and the NLR. For all grids that are detected with an abnormal NLR change $24 \mathrm{~h}$ before and after the typhoon, the two-sided Mann-Whitney U-test was used to examine whether the difference in the component $T_{1}+e_{1}-T_{2}$ of the NLR time series before (i.e., 13 and 14 September) and after (i.e., 18 and 19 September) the typhoon is statistically significant ( $p$-value $<0.05)$. It is more challenging to effectively detect light loss after a hazard in the grids normally with a low NTL value due to the background noise [13]. To address this issue, we selected a NTL radiance threshold based on the difference in the probability density function (PDF) of the NTL values between the grids with and without a stable NLR.

We also checked whether the dNTL in each grid was caused by the change of background signal before and after the typhoon. In this study, the background NTL signal is defined by the radiance in the grids with no ambient population, which were identified from both of the 2016 and 2017 LandScan rasters. The water grids were then masked using the CCI-LC 2015 dataset. In total, we found 2309 of such grids and the mean and standard deviation (SD) of their dNTL values are 0 and 0.15 , respectively. In the subsequent analyses, we excluded all the grids with a dNTL value greater than -0.15 and less than 0.15 as it is very likely that such a low dNTL value might be attributed to the change of background signal rather than the typhoon. The dNTL rasters were then resampled to the same resolution as of the cNLR rasters using the nearest-neighbor resampling method. 
We then constructed a confusion matrix to evaluate whether the NTL signals can be used to identify the grids with significant variations in the NLR. As shown in Figures 5 and 6, a confusion matrix contains four elements, including false positives (FP, a negative observation is classified to be positive), false negatives (FN, a positive observation is classified to be negative), true positives (TP, a positive observation is classified to be positive), and true negatives (TN, a negative observation is classified to be negative). The precision $(\mathrm{TP} /(\mathrm{TP}+\mathrm{FP}))$ is the proportion of the positive samples that are correctly classified to the total number of the samples that are classified to be positive. The fallout $(\mathrm{FP} /(\mathrm{FP}+\mathrm{TN}))$ is the ratio between the number of negative samples that are not correctly classified to the total number of negative samples. The miss rate $(\mathrm{FN} /(\mathrm{FN}+\mathrm{TP}))$ is the proportion of the positive samples that are incorrectly classified. The recall $(\mathrm{TP} /(\mathrm{TP}+\mathrm{FN}))$ is the proportion of the positive samples that are correctly classified to the total number of positive samples. The accuracy $((\mathrm{TP}+\mathrm{TN}) /(\mathrm{TP}+\mathrm{TN}+\mathrm{FP}+\mathrm{FN}))$ is defined as a ratio between the correctly classified samples to the total number of samples and is used to measure classification performance. The F1 score $(2 \times($ precision $\times$ recall $) /($ precision + recall $))$ is the harmonic mean of the precision and the recall. A high F1 score would indicate a more optimal classification performance.

(a)

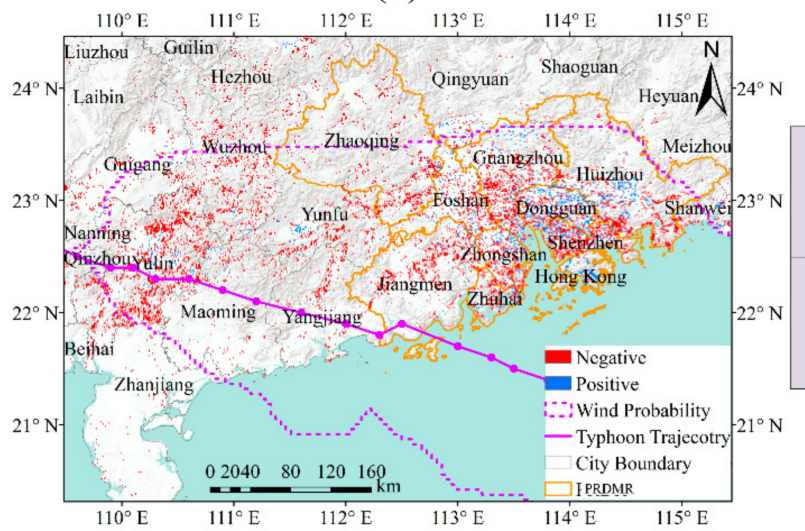

(c)

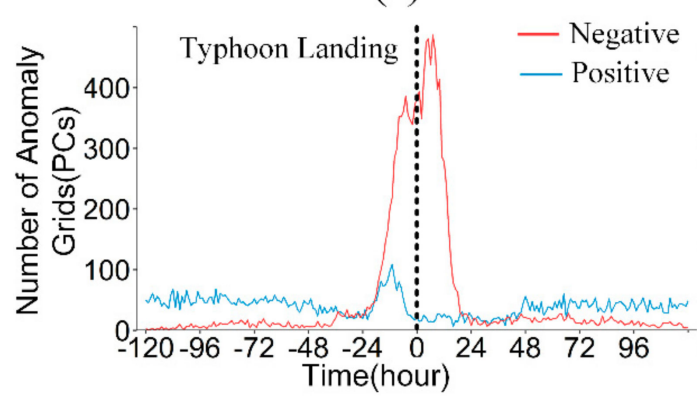

(b)

\begin{tabular}{|c|cc|}
\cline { 2 - 3 } \multicolumn{1}{c|}{} & $\begin{array}{c}\text { Abnormal } \\
\text { Grid }\end{array}$ & $\begin{array}{c}\text { Normal } \\
\text { Grid }\end{array}$ \\
\hline Within the & 8921 & 20955 \\
extent of & $72.9 \%$ & $29.5 \%$ \\
50 knot wind & $(\mathrm{TP})$ & $(\mathrm{FP})$ \\
\hline Outside the & 3323 & 50021 \\
extent of & $27.1 \%$ & $70.5 \%$ \\
50 knot wind & $(\mathrm{FN})$ & $(\mathrm{TN})$ \\
\hline \multicolumn{2}{c|}{ TP:True positive,FP:False positive } \\
FN:False negative,TN:True Negative \\
Total number of samples(TS):83220
\end{tabular}

(d)

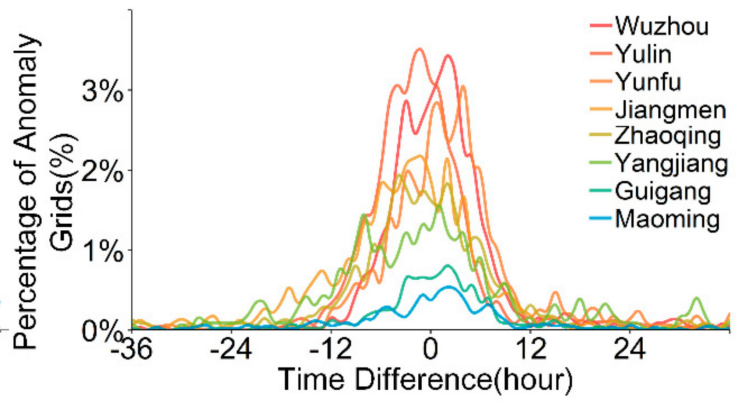

Figure 5. (a) The anomaly grids detected when Typhoon Mangkhut is landing. (b) The confusion matrix of the abnormal and normal grids within and beyond the storm-force wind impacting zone. (c) Temporal variation in the number of abnormal grids within our study area, and (d) the percent and number of anomaly grids at the city level. 
(a)

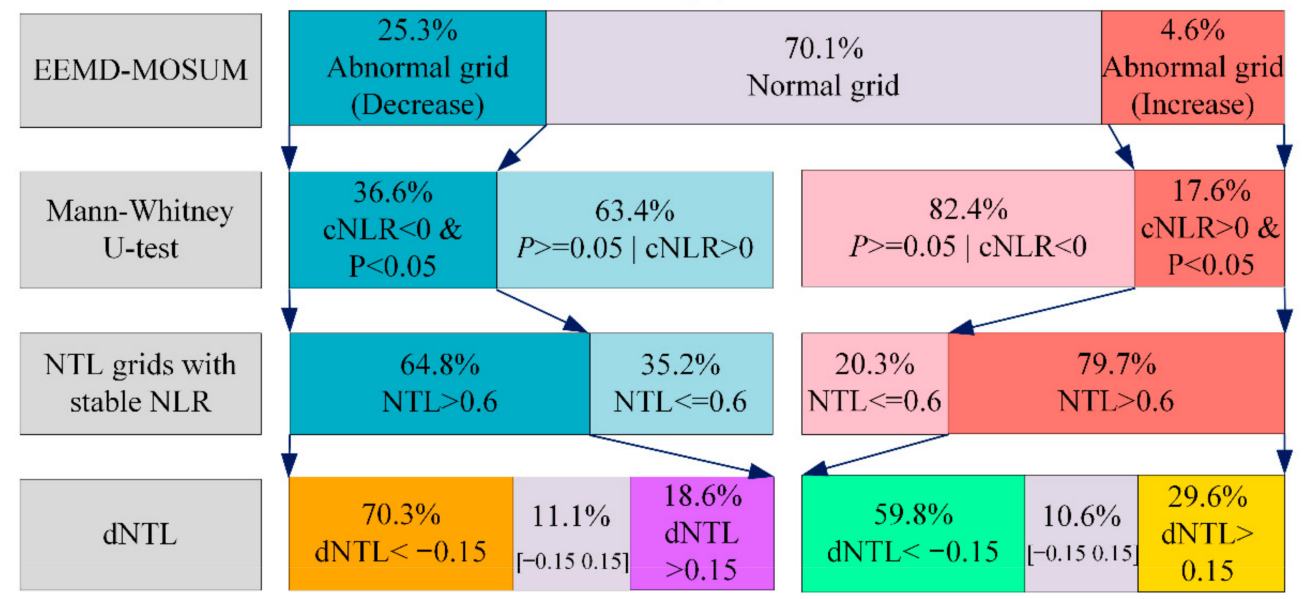

(b)

(c)

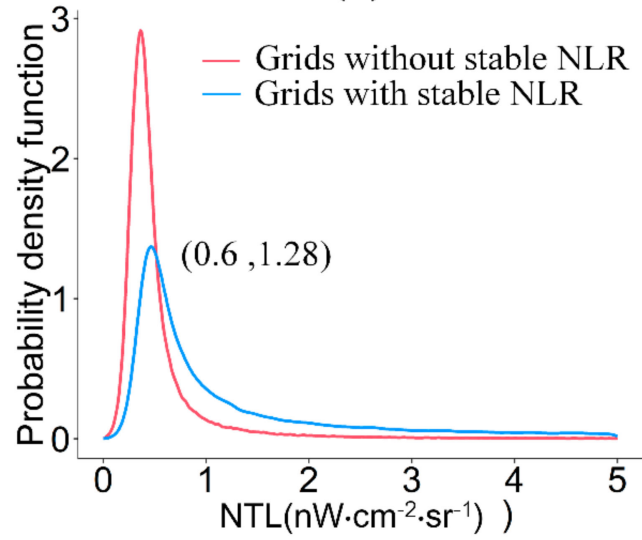

\begin{tabular}{|r|c|c|}
\cline { 2 - 3 } \multicolumn{1}{c|}{} & $\begin{array}{c}\mathrm{cNLR}<0 \\
\text { and } P<0.05\end{array}$ & $\begin{array}{c}\mathrm{cNLR}>0 \\
\text { and } P<0.05\end{array}$ \\
\hline $\mathrm{dNTL}<-0.15$ & 1260 & 113 \\
& $91.8 \%$ & $8.2 \%$ \\
$(\mathrm{TP})$ & $(\mathrm{FP})$ \\
\hline $\mathrm{dNTL}>0.15$ & 337 & 56 \\
& $85.8 \%$ & $14.2 \%$ \\
TP:True positive,FP:False positive \\
FN:False negative,TN:True Negative \\
Total number of samples(TS):1766
\end{tabular}

Figure 6. Grid-level consistency between the dNTL and cNLR. (a) The relative proportions of different types of grids with various cNLR and dNTL values. (b) The NTL radiance threshold selected according to the differences in the PDF of the NTL values between grids with/without stable NLR. (c) The confusion matrix that was used to distinguish grids with significantly decreased NLR based on the dNTL values.

Due to the unequal variances and unequal sample sizes, we used Welch's two-sample $t$-test to test whether the means of the dNTL values between the grid groups with different cNLR values are equal or not. Quantile regression were used to study the quantitative relationships between the dNTL and cNLR at both the grid and county levels. We categorized the county-level total and average magnitudes of the dNTL and cNLR into three classes separately using the Jenks natural breaks classification method, which clusters data into different classes by seeking minimum variance within a class and maximum variance between classes [34]. In total, there are nine different combinations between the total and average magnitudes of the dNTL and cNLR, respectively. We then examined the spatial differentiation between the county-level declines among the different dNTL and cNLR groups. The GWR [55] is used to analyze county-level relationships between total/average dNTL and cNLR on the log scale. We used the adaptive distance approach to determine the spatial context (the Gaussian kernel) and the corrected Akaike Information Criterion (AICc) method to select the optimal bandwidth for the GWR analysis. The Gaussian kernel for GWR procedure is constructed as an adaptive distance. Finally, the total and average losses of the cNLR and dNTL by county were calculated for all 220 counties within our study area, and the quantile regression methods were again used to quantify the relationships between the total regional declines of the dNTL and cNLR and the damage statistics at the county level, respectively. 
We used ENVI version 5.5 (Exelis Visual Information Solutions, Boulder, CO, USA) and MATLAB and Statistics Toolbox 2015a (MathWorks, Inc., Natick, MA, USA) to pre-process the data and identify the stable grids. The EEMD-MOSUM procedure was built using the Fast EEMD package [56] in Matlab and the strucchange package in R $[57,58]$. Quantitative relationships between the NLR and NTL signals at the grid and county levels, as well as between the dNTL/cNLR and disaster damage estimates, were completed in stats and the quantreg packages in R [57]. We performed the GWR analysis in ArcGIS Desktop 10.2 (Environmental Systems Research Institute, Redlands, CA, USA).

\section{Results}

\subsection{Quantitative Associations between the NLR and Typhoon Mangkhut and between the NLR and} NTL Signals

\subsubsection{Quantitative Associations between the NLR and Typhoon Mangkhut}

Variations in human activities as reflected by NLR data are closely associated with the evolution of the typhoon (Figure 5). Spatially, abrupt NLR changes were detected in 12,244 grids, accounting for $14.7 \%$ of the total 83,230 stable grids within our study area. In total, 8921 out of the 12,244 grids are located within the 50-knot wind impacting zone. The accuracy of $71.8 \%$ in the confusion matrix (Figure $5 b$ ) indicates that the grids within the wind impacting zone are more significantly affected by the typhoon. Temporally, the percent of anomaly grids around a weather station is very similar to that of the anomaly grids within the 50-knot wind impacting zone (Figures S3 and S4). It starts to sharply increase when the typhoon is approaching, peaks when the typhoon hit the station, and then sharply drops to a low value after that (Figure 5c). It is negatively and positively correlated with the air pressure (average $r=-0.88$ ) and wind speed (average $r=0.73$ ), respectively. The numbers of positive and negative anomaly grids peak 12 and five hours before and after the typhoon made landfall, respectively. The positive anomaly grids are mainly clustered in the PRDMR, including the cities of Hong Kong, Dongguan, Shenzhen, Huizhou, and Zhongshan (Figure 5b). These results are consistent with our previous work in quantifying human activities in response to Typhoon Hato, indicating that positive anomalies are more likely to be trigged by human activities in response to the early warning signals before typhoon landing, and people in the PRDMR are more sensitive to the early warning signals. The peak percent of anomaly grids is much higher in the cities that are closer to Mangkhut's track (e.g., Wuzhou, Yulin) and lower in those distant cities (e.g., Zhaoqing, Guigang). When the typhoon struck a city, the anomaly grid percent exploded and then dropped significantly after the typhoon passed by (Figure 5d).

\subsubsection{Quantitative Associations between the dNTL and cNLR at the Grid Level}

Figure 6a and Figure S5 show the relative proportions and numbers of the grids with different dNTL and cNLR values within the 50-knot wind impacting zone. A total of 29,876 NLR grids were identified as stable grids within the impacting zone, out of which $7574(25.3 \%)$ shows abnormal decreased and 1337 (4.6\%) abnormal increased NLR values, respectively. As shown by the Mann-Whitney U-test results (Table S1), after the typhoon made landfall, $36.6 \%$ of the 7574 grids keep showing decreased NLR values whereas the remaining $63.4 \%$ shows increased NLR values. By contrast, $17.6 \%$ of the 1337 grids keeps showing an increased NLR value after the typhoon made landfall and the remaining $82.4 \%$ shows a decreased NLR value. The results jointly indicate that nearly $33.7 \%$ of abnormal grids during the typhoon landing has not returned to the normal levels in nearly two days after Typhoon Mangkhut.

The grids were further investigated based on their NTL values. A NTL threshold of $0.6 \mathrm{nW} \cdot \mathrm{cm}^{-2} \cdot \mathrm{sr}^{-1}$ was used to extract the grids with a stable NLR (Figure $6 \mathrm{~b}$ ). When the NTL values are higher than the threshold, the PDF values in the stable grids tend to be higher than those in the non-stable grids. In other words, grids are more likely to have a stable NLR when their NTL values are higher than $0.6 \mathrm{nW} \cdot \mathrm{cm}^{-2} \cdot \mathrm{sr}^{-1}$. Approximately $64.8 \%$ of the grids with an abnormally-decreased NLR value has a NTL value higher than $0.6 \mathrm{nW} \cdot \mathrm{cm}^{-2} \cdot \mathrm{sr}^{-1}$. Out of these grids, approximately $70.3 \%$ 
and $18.6 \%$ of them show a declined and increased NTL brightness value, respectively. By contrast, approximately $79.7 \%$ of the grids with an abnormally-increased NLR value has a NTL value greater than $0.6 \mathrm{nW} \cdot \mathrm{cm}^{-2} \cdot \mathrm{sr}^{-1}$. About $59.8 \%$ and $29.6 \%$ out of these grids show a declined and increased NTL brightness value, respectively. The results indicate that nearly $69.3 \%$ of the anomaly NLR grids can be inferred from the declined NTL brightness across our study area. The calculation of the percentage of $33.7 \%$ and $69.3 \%$ can be found in Supplementary Materials.

The precision of 0.92 shows that over $90 \%$ of the grids with a dNTL value less than -0.15 shows a significant decrease in the NLR (Figure 6c). The fallout of 0.67 shows that about $67 \%$ grids with a significantly increased NLR shows reduced NTL signals, mainly due to the typhoon-related events such as emergency resettlement, disaster relief, and the spread of situational awareness and information through the social networks. The miss rate of 0.21 shows that approximately $21 \%$ of grids with a significantly decreased NLR are unlikely to be sensed by the NTL signals. This is probably due to some disadvantages of the NTL images, such as the rough spatial resolution, the effects of nocturnal airglow to the grids with lower radiance value, along with the data issues caused by image resampling and projection, and the complex effects of anthropogenic lighting on the NTL. Overall, a 0.85 F1 score and a 0.75 accuracy jointly suggest that the NLR is closely associated with the daily NTL signals when our study area was impacted by Typhoon Mangkhut.

\subsection{Relationships between the cNLR and $\mathrm{ANTL}$ at the Grid and County Level}

\subsubsection{Relationships between the cNLR and dNTL at the Grid Level}

Figure 7a shows notable difference in the dNTL between the grid groups with different cNLR values. In this study, we first divided the grids with a significant declined NTL brightness value (i.e., dNTL $<-0.15$ ) into two datasets based on whether or not the grids showed a significant decreased NLR values before and after the typhoon. The probability densities of the two datasets both showed a right-skewed and long-tailed distribution, due to highly uneven decline in the NTL brightness in response to the impact of Typhoon Mangkhut. Moreover, the medians of the two datasets $(-1.2$ and -1.7 for the grids with $c N L R<0$ and cNLR $>0$, respectively) indicate relatively limited NTL brightness loss in most grids with a significantly decreased NLR value. The mean of the dNTL for all grids with a significant decreased NLR values is larger than the others and the difference between the means of the two datasets are statistically significant as shown by Welch's two-sample $t$-test $(\mathrm{t}=5.3717, \mathrm{df}=3365.5$, $p$-value $<0.001)$. These results suggest that the significant decreased human activities could be sensed by relatively limited loss of NTL brightness. Overall, the grids with significant decreased human activities tend to show a declined NTL brightness value.

There is likely a log-linear correlation between the dNTL and cNLR in the 1260 grids with a remarkable decline of both the NTL and NLR (Figure 7b). The Pearson correlation coefficient (Pearson' $r=0.44, p$-value $<0.01)$ indicates an average positive correlation at the log-linear scale between the $\mathrm{dNTL}$ and abnormally-decreased NLR. The quantile regression results (Figure $7 \mathrm{~b}$ and Table S2) show that the slope coefficient estimates of the cNLR gradually increase from the lower quantiles to the higher quantiles $(0.3,0.38,0.48$ for $0.25,0.50,0.75$ quantiles, $p$-value $<0.01)$, implying that the increase in the dNTL significantly corresponds to the increased dispersion of the cNLR. In other words, the decline rate of the NTL brightness gradually increases from the less-lit grids to the better-lit grids with respect to the decline magnitude of human activities. Similarly, although only $0.8 \%$ of grids shows an increased NLR value after the typhoon, an average positive correlation (Pearson' $\mathrm{r}=0.4$, $p$-value $<0.01$ ) was found between the dNTL and cNLR across the 113 grids with a declined NTL and a significantly increased NLR value (Figure 7c). The quantile regression results (Figure 7c and Table S2) also suggest a notable dispersion $(0.41,0.54,0.64$ for $0.25,0.50,0.75$ quantiles, $p$-value $<0.01)$ of the log-linear relationship between the dNTL and cNLR among the 113 grids. These results suggest abnormally increased/decreased human activities are positively correlated with the dimmed NTL signals at a log-linear scale. 


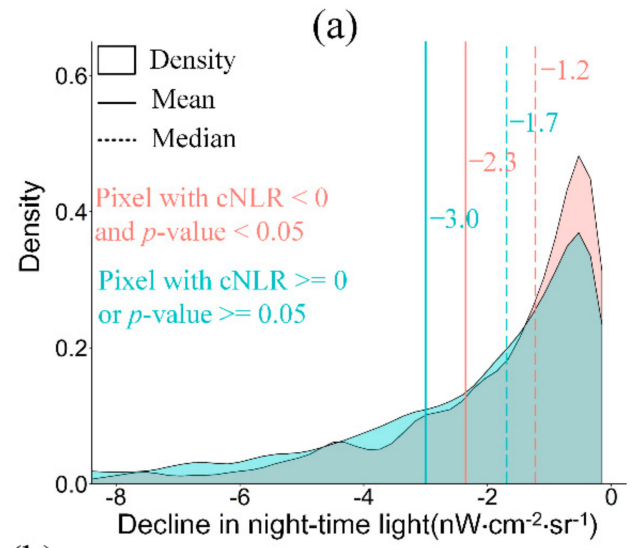

(b)
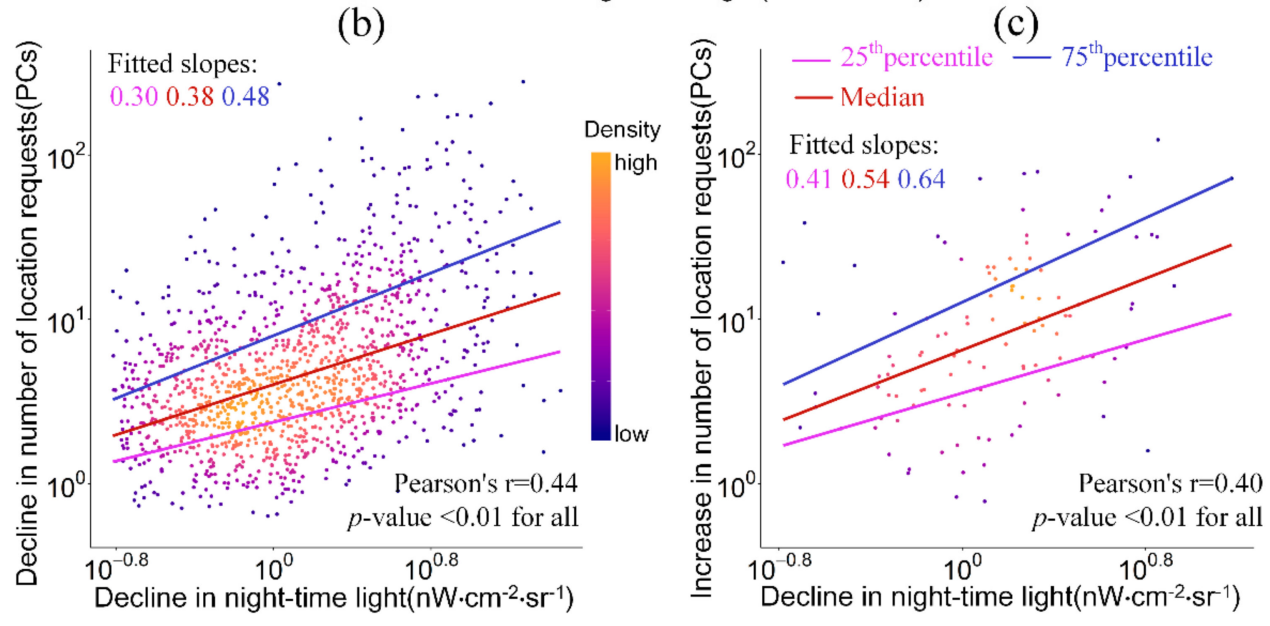

Figure 7. Grid-level relationships between the dNTL and cNLR. (a) The probability density distribution curves of the dimmed NTL grids with a reduced or an increased NLR after the typhoon. $(\mathbf{b}, \mathbf{c})$ Relationships between the dNTL and cNLR at the grid level quantified by the quantile regression.

\subsubsection{Relationships between the cNLR and dNTL at the County Level}

Positive correlations also exist between the dimmed NTL signals and the significant changes of the NLR at the county level. We only analyzed the 101 counties with the casualty and damage statistics. The 0.52 Pearson correlation coefficient $(p$-value $<0.001)$ indicates that a moderate positive log-linear relationship may exist between the total dNTL and cNLR at the county level (Figure 8a). A stronger positive correlation is found between the county-level average dNTL and cNLR (Pearson's $\mathrm{r}=0.77, p$-value $<0.001$ ) (Figure $8 \mathrm{~b}$ ). The relatively weaker correlation between the county-level dNTL and cNLR could be attributed to the differences in population and economy development levels among the cities. For example, Zhongshan City (population: 3.26 million) and Beiliu City (population: 1.51 million) have very similar total cNLR but significant different total dNTL values. The GDP of these two cities is $\$ 54.9$ and $\$ 4.7$ billion as of 2018 , respectively. The stronger correlation between the means of the dNTL and cNLR suggests similar influences of the typhoon on the dNTL and cNLR at the county level. As shown in Figure 8a,b and Table S3, the quantile regression results (including the $0.25,0.50$, and 0.75 quantiles) also show log-linear relationships between the dNTL and cNLR in terms of their total and average regional values but in totally opposite trends. The total regional NTL brightness gradually drops at a slower pace (i.e., a rate from 0.73 to 0.49 ) from less- to better-lit grids. By contrast, the average regional NTL brightness also drops but at a faster pace (i.e., with a rate from 0.66 to 0.91 ) from the less- to better-lit grids. 


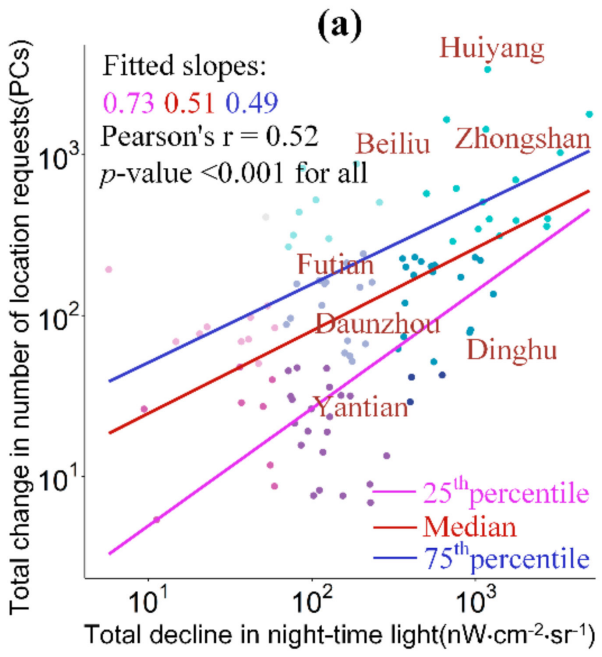

(c)

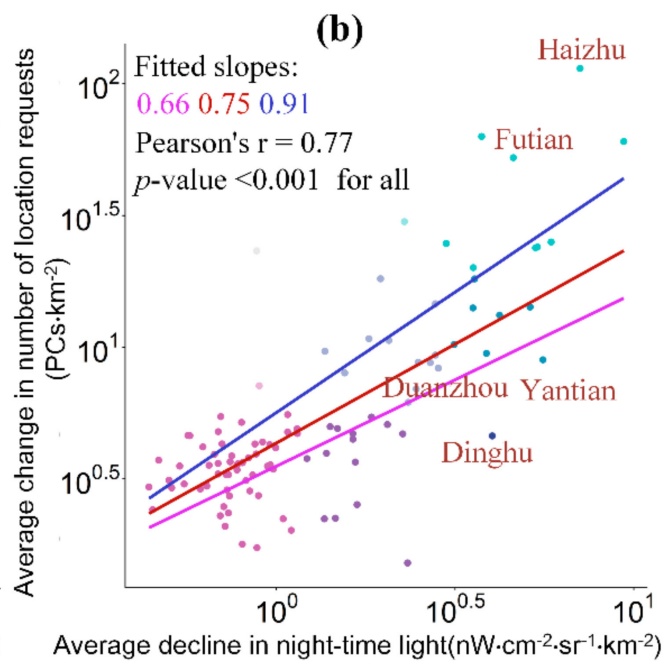

(d)

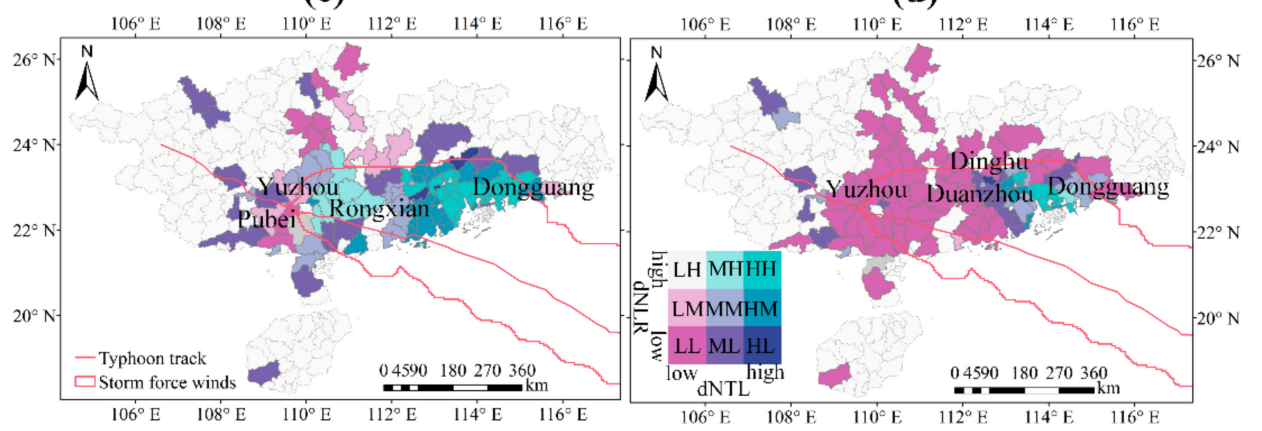

Figure 8. $(\mathbf{a}, \mathbf{b})$ The log-linear relationships between the total and average magnitudes of the dNTL and cNLR. (c,d) Spatial differentiation between the total and average magnitudes of the dNTL and cNLR that were separately divided into three categories.

The associations between the dimmed NTL signals and cNLR at the county level also exhibit a significant pattern across our study area (Figure $8 c, d$ ). For the total magnitude of the dNTL and cNLR, most counties in the core area of PRDMR are categorized into type $\mathrm{HH}$, and surrounded by the type HM counties. Counties located on the north side of the typhoon track mainly fall into types $\mathrm{MH}$ and MM, whereas those on the north side into types LL, LM, and ML, indicating the different impacts of the typhoon on the cities on the north and south sides of the track.

For the average magnitude of the dNTL and cNLR, only $16.8 \%$ of the counties falls into types HM, $\mathrm{MH}$, or $\mathrm{HH}$, which are mainly clustered in the densely populated areas of PRDMR. By contrast, type LL counties account for $54.4 \%$ of all the 101 counties. These results indicate that the variance of the average magnitude of the dNTL and cNLR in most counties is less than that of their total magnitudes. In particular, the Yuzhou district in Yulin City falls into type ML and is surrounded mainly by type LL counties. Similar patterns were also observed in Dinghu and Daunzhou Districts in Zhaoqing City, indicating that the NTL signals in these districts are more sensitive to the impacts of the typhoon.

The GWR analysis results further reveal the spatial patterns of the county-level connections between the dNTL and cNLR on a log scale. The global adjusted $R^{2}$ values (Table S4) indicate that $\sim 26 \%$ of the inter-county variations in the total changes of human activities can be explained by the total brightness NTL loss, and $~ 59 \%$ of the inter-county variations in the average NLR can be explained by the average NTL brightness loss. As shown in Figure 9, relatively higher local $R^{2}$ and positive local coefficients between the total dNTL and cNLR (i.e., a closer association between these two variables) were mainly found in the cities located in the north of the typhoon track, including Huizhou, Dongguan, Shenzhen, Zhuhai, Zhongshan, Guangzhou, Guigang, and Wuzhou (Figure 9a,b). Relatively higher local $R^{2}$ values and positive local coefficients between the average dNTL and cNLR (i.e., a closer 
association between these two variables) are mainly found in the PRDMR cities and Qingyuan, a city in northern Guangdong (Figure 9c,d). By contrast, negative local coefficients between the total dNTL and cNLR were mainly found in the cities located in the south or the end of the typhoon track, especially for the more inland cities such as Qingzhou, Fangchenggang, and Nanning. Negative local coefficients between the average dNTL and cNLR were mainly found in Guilin city, which is far away from the typhoon track. These results are probably due to the different recovery speeds of the human activities and NTL signals, as well as the different impact intensities of Mangkhut in these areas. Figure S6 shows the GWR standardized residuals of six counties seriously deviate from the model (absolute value of deviation $>2.5$ ). The six counties are located beyond or near the storm-force wind impacting zone. This indicates that human activities or the NLR signals have been severely affected in these areas, probably due to the poor disaster-bearing capacity of these six counties. In short, the above results, on the one hand, provide a fundamental measure of the regional-level connection between the NTL data and the human activities and, on the other hand, reveal the diversity of the two signals in response to the typhoon.

(a)

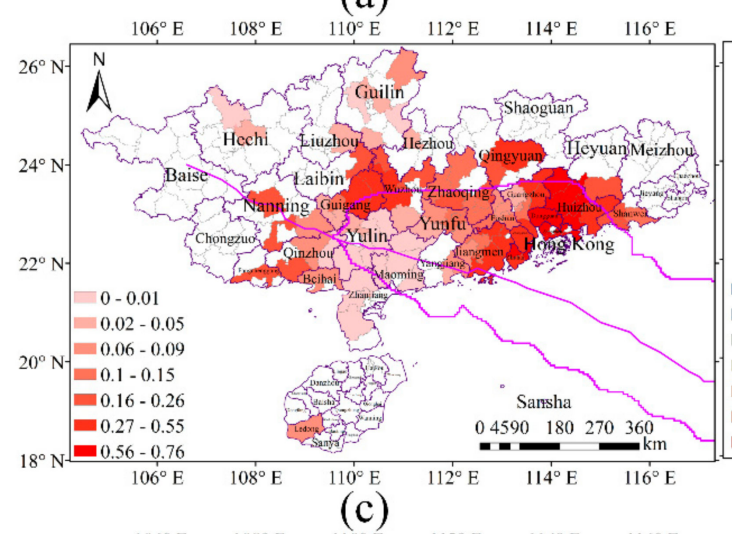

(b)

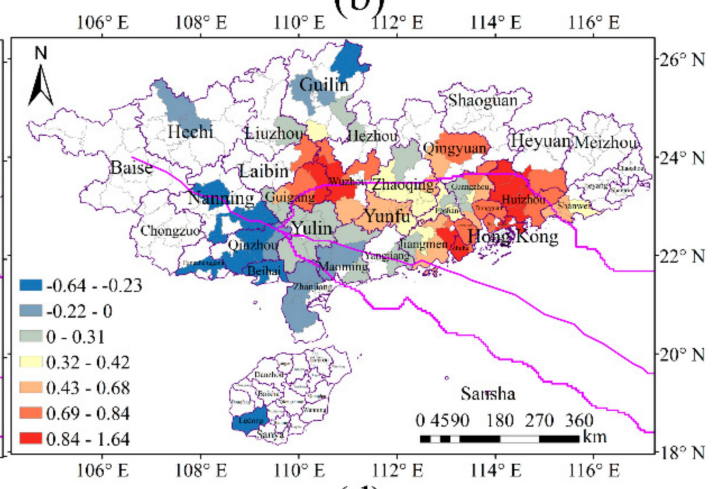

(d)

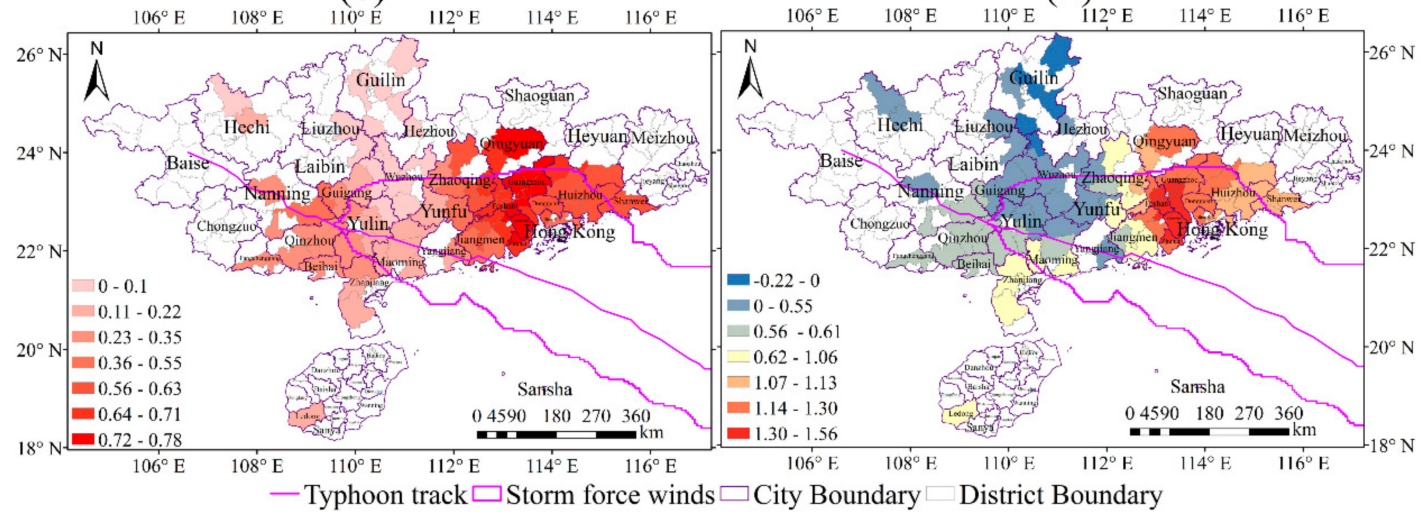

Figure 9. $(\mathbf{a}, \mathbf{b})$ The local $\mathrm{R}^{2}$ values and the local coefficients from the GWR model, in which county-level total dNTL and cNLR on the log scale are the dependent and explanatory variables, respectively. (c,d) The local $\mathrm{R}^{2}$ values and the local coefficients from the GWR model, in which county-level average dNTL and cNLR on the log scale are the dependent and explanatory variables, respectively.

\subsection{Relationships between the dNTL and cNLR in Disaster Damage Estimates}

We then examined the associations between the damage estimates against the total magnitudes of the dNTL and cNLR at the county level (Figure 10 and Table S5). The Pearson's r in Figure 10a-c indicates log-linear moderate positive correlations between the county-level cNLR and the damage estimates. Although only $24.6 \%$ of the grids with abrupt changes remains abnormal after the typhoon, moderate positive correlations were found between the county-level dNTL and damage estimates at the log-linear scale (Figure 10d-f). This indicates that Tencent's location-aware data can be used to estimate 
typhoon-induced damages both during and after the typhoon. The significant dispersions in quantile regressions reveal non-uniform relationships between the cNLR and damage estimates. Quantile regression results also show the dispersion differences between the cNLR and damage estimates before and after the typhoon. For example, we found a more obvious dispersed log-linear relationship between the cNLR and the direct economic loss after the typhoon (from 1.28 to 0.69) than that during the typhoon (from 0.79 to 0.60 ). These results suggest that inter-county associations between the cNLR and damage estimates may change over time due to the different recovery rates of human activities in different cities.

(a)

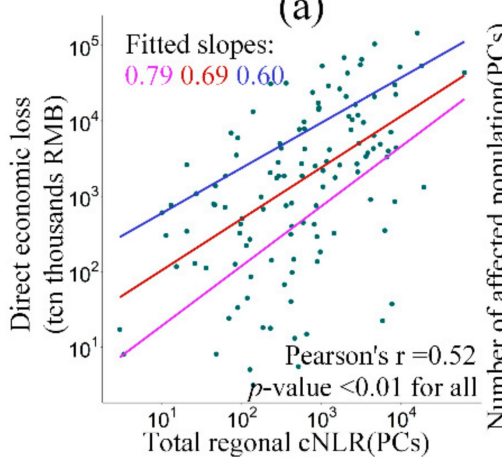

(d)

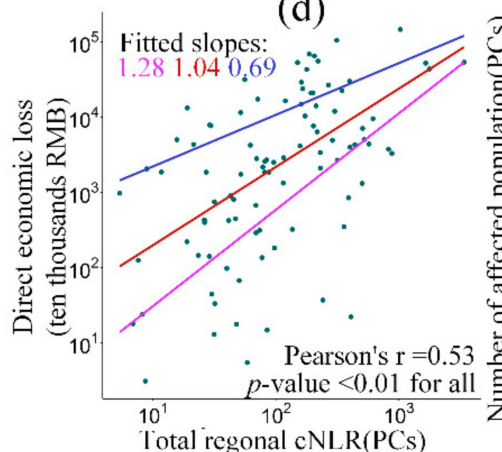

(g)

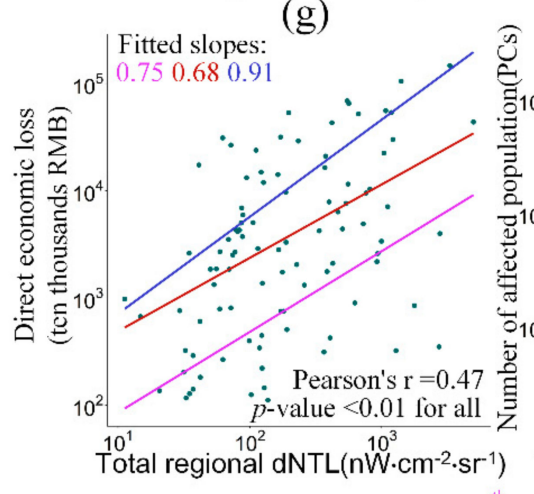

(b)

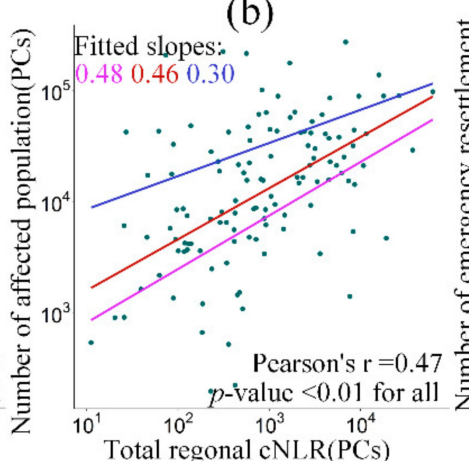

(e)

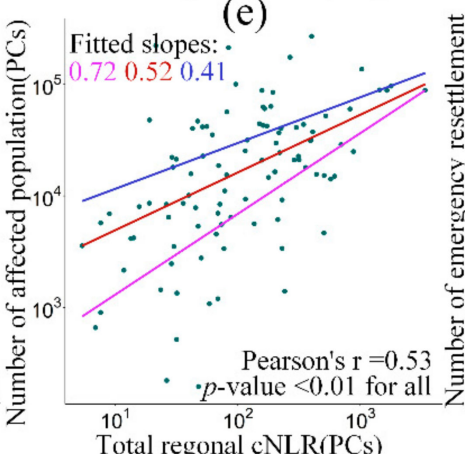

(h) (c)

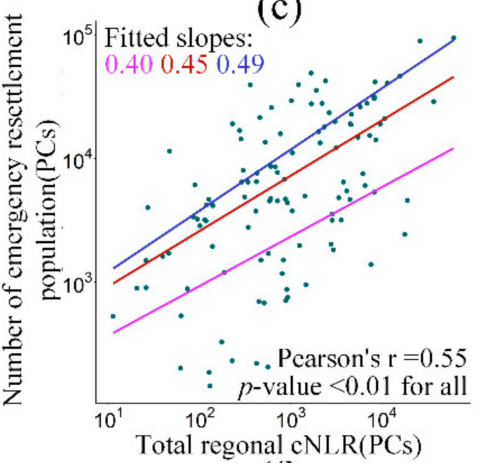

(f)

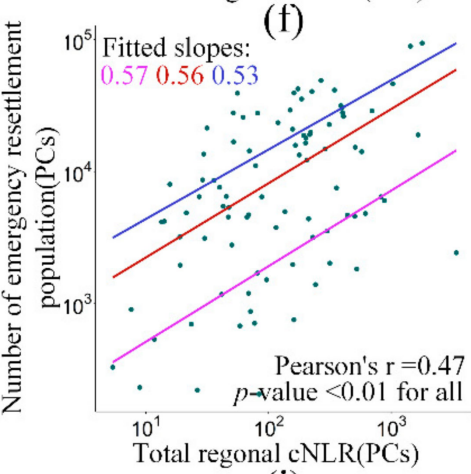

(i)

$$
\text { - } 25^{\text {th }} \text { percentile }- \text { Median }-75^{\text {th }} \text { percentile }
$$

Figure 10. (a-c) The log-linear relationships between the county-level cNLR and damage estimates during the typhoon. (d-f) The log-linear relationships between the county-level cNLR and damage estimates two days after the typhoon. (g-i) The log-linear relationships between the county-level dNTL and damage estimates two days after the typhoon.

There is a moderate positive correlation (Figure 10i) between the $\mathrm{dNTL}$ and emergency resettlement population (Pearson's $r=0.5, p$-value $<0.01$ ); and a weaker correlation (Figure $10 \mathrm{~g}$ ) between the dNTL and the direct economic losses (Pearson's $r=0.47, p$-value $<0.01$ ), as well as between the dNTL and the number of affected population (Pearson's $r=0.32, p$-value $<0.01$ ). The results clearly show that the NTL signal is not as good as the NLR in explaining the direct economic losses and the number of 
affected population. This probably is due to the own characteristics of these two datasets. The NTL signals only change when an area is hit by power outages and significant infrastructure damages. By contrast, Tencent's location-aware data can capture disrupted human activities that may be caused by a variety of disaster-induced problems that are not limited to power outages and infrastructure damages. In short, the above results show that there are statistically significant log-linear correlations between cNLR and damage estimates and between dNTL and damage estimates at the county level, and also reveal the difference between the NTL signals and the NLR data in sensing the loss and damages caused by the typhoon.

\section{Discussion}

\subsection{Quantitative Associations between the NLR and Typhoon Mangkhut and between the NLR and NTL Signals}

Our study showed that a typhoon like Mangkhut could significantly impact human activities, which could be inferred from the changes of Tencent's location data and the daily NTL signals. Our previous work [48] also shows Typhoon Hato had a significant impact on the human activities at grid and city levels. Typhoon Hato and Mangkhut both caused significant spatiotemporal fluctuations of the NLR. The damages they caused are moderately and positively correlated with the changes of the NLR. The differentiation between the north and south of the typhoon track were found in the recovery duration of human activities during Typhoon Hato. Similar differentiation along the typhoon track was also found in the county-level associations between the dimmed NTL signals and cNLR during Typhoon Mangkhut. The results suggest that Tencent's data could be used as a proxy for measures of the impacts of different typhoons. High spatial consistency between the NLR and NTL signals further showed that the NLR was closely associated with the daily NTL signals after Typhoon Mangkhut, implying that the daily NTL signals could also be used to evaluate the impacts of the typhoon.

\subsection{Relationships between the cNLR and dNTL}

This study shows the change of the daily NTL brightness is correlated with the change of NLR at both the grid and county levels during Typhoon Manghut. Previous studies have found significant spatial consistency between the monthly dNTL and the significantly decreased NLR across 332 Chinese cities during the Chinese New Year holiday [46]. The daily dNTL is also associated with the density of power-related tweets in the areas affected by Hurricane Sandy [26]. There is a log relationship between the monthly stable NTL brightness and the NLR at the provincial, city, and county levels [47], as well as between the dNTL and significantly decreased NLR at both the grid and city levels in China [46]. In short, the NTL and NLR signals are strongly associated with human activities at multiple temporal and spatial scales. However, the quantitative relationships between the NTL and NLT may vary significantly at different spatiotemporal observation scales.

The relationships between abnormal human activities and the dimmed NTL signals, as well as the impacts of the typhoon on human activities, are affected by various factors at grid and city level. Firstly, the decreased human activities could be sensed by relatively limited loss of the NTL brightness probably due to two reasons. The first is probably because the typhoon track mainly passed through the rural and suburban areas in Yangiiang, Maoming, and Yulin. Grids in these areas usually have relatively low NTL radiance and so that a narrow range of the possible brightness loss. The second reason may be because both human activities and the NTL radiance in the rural and suburban areas are more prone to be affected by the typhoon. Secondly, approximately $10 \%$ of the grids keep showing a decreased NLR value after the typhoon. This is probably due to the areal difference of the recovery speeds and the uneven impacts of the typhoon. Only less than $1 \%$ of the grids keeps showing an increased NLR value after the typhoon, which could be attributed to the increased NLR that results from typhoon-related events such as emergency resettlement, disaster relief, and the spread of situational awareness and information through the social networks. Results from this study clearly 
show the typhoon's impacts on human activities, which could be inferred from the change of the NLR. The abnormal human activities are associated with the dimmed NTL signals at the grid level as shown by the average positive correlations at a log-linear scale. Thirdly, the variations in the relationships between the county-level dNTL and cNLR could also be affected by the distance from the county to the typhoon's track, as well as the counties' socioeconomic development levels.

\subsection{Integration of Remote Sensing and Social Media Data for Improving Emergency Response}

Our study also shows the NTL and NLR signals are complimentary in sensing the changes of human activities in response to Typhoon Mangkhut, as well as the typhoon-induced damage. Disasters like hurricanes and floods may trigger immediate short-term power outages, which may recover in a few days [13]. Such disasters are frequently accompanied with heavy rainfall and massive cloud coverage, which make it impossible to detect power outages from the daily NTL signal. Fortunately, the NLR is not as significantly affected by the weather as the NTL. In such a scenario, the NLR may serve as a potential alternative when the NTL signal is missing as average log-linear positive correlations are found between the two signals. On the other hand, NTL can directly sense disaster-induced power outages, which may affect the NLR but could not be accurately inferred from the change of the NLR.

As shown in this study, the integration of remote sensing and social media data shows its advantages in evaluating disaster-induced impacts and damages [26,27,30,32]. On the one hand, social media data can be utilized to bridge the temporal gaps in the satellite-based observations. On the other hand, it can be fused into the satellite images to significantly improve the spatial resolution of disaster assessment results [33]. Before developing or improving the methods that could be used to integrate the two datasets, it is essential to thoroughly examine how the remote sensing and social media data are associated at multiple spatial levels as in this and some previous studies [15,59]. Furthermore, some technical and methodological challenges in integrating social media and remote sensing data are needed to be addressed in the future. First, the demographic biases and spatiotemporal sparsity may pose a challenge in using social media data in disaster evaluation at fine spatiotemporal scales over a large geographical space, especially in sparsely populated areas. Second, there is a need of more comprehensive frameworks or methods for exploring the complex relationship between disasters and human activities, better integrating and exploiting multiple-source data in disaster investigation [59]. However, extraction of disaster-related information from social media is challenging due to a wealth of information, as well as the unstructured and heterogeneous nature in the social media data [15].

\subsection{Uncertainties}

Various uncertainties in the data may introduce biases that could influence the correlations we found in this study, including those between the changes of the dNTL and cNLR, and between the typhoon-induced damage estimates and the changes of the NTL and NLR. First, the NLR may be overestimated and underestimated in densely- and less-populated, respectively. The NTL in sparsely-populated areas tends to be significantly affected by the rescue lights after the disaster due to the low normal NTL brightness and limited disaster-induced light loss. In contrast, there would be very limited influences of the rescue lights on the NTL in well-lit densely-populated areas as light loss in such areas is much more significant [13]. Second, errors may be introduced when we corrected the influences of the atmosphere, terrain, and vegetation on the daily NTL signal [6]. Spatial resampling, the over-glow effect, the usage of lighting technology, and the complexity of underlying drivers such as the distribution of residential sites, may jointly introduce more bias to the NTL signals [60]. Third, the correlation between damage estimates and total regional magnitudes of $\mathrm{dNTL} / \mathrm{cNLR}$ could be further undermined by the inconsistency between loss statistics and human activity dynamics sensed by the NTL and NLR signals. For example, it is really challenging to use the NTL and NLR signals to sense the direct economic losses in sectors such as agriculture, forestry, fishery, and water conservancy due to the lack of relatively stable human activities and consequently low NTL and NLR. Furthermore, loss and damage statistics collected by the grassroot administrative departments in China often suffer problems 
such as the lack of unified statistical standards, time lags, duplicated calculations, and exaggeration of losses, which would further add bias to the correlations between the total regional magnitudes of the $\mathrm{dNLT} / \mathrm{cNLR}$ and damage estimates.

\section{Conclusions}

The NPP-VIIRS DNB daily data have been used as a proxy measure for detecting and assessing hazard-induced power outages, losses and damages. Previous studies have shown that changes of human activities could be inferred from variations in the NLR. In this study we investigated whether the DNB-derived NTL daily signals respond in a timely manner to the geo-tagged human activity dynamics at the grid and county levels when Typhoon Mangkhut inflicted upon Southern China.

The main findings of this study include: (1) the abnormal geo-tagged human activities are closely associated with the impacts of Typhoon Mangkhut. The geo-tagged human activities in the grids within the storm-force-wind impacting zone are more significantly affected by the typhoon than those beyond. Typhoon Mangkhut's impacts on the geo-tagged human activities are manifested by the influences of air pressure and wind speed on the number of location-service requests. More grids show reduced location-service request when the wind is stronger and air pressure is lower around the nearby weather stations. (2) Approximately $69.3 \%$ of the grids with reduced location-service request also shows declined NTL brightness nearly two days after Typhoon Mangkhut. Overall, the F1 score of 0.85 and the accuracy of 0.75 in the confusion matrix jointly suggest that the geo-tagged human activities is closely associated with daily NTL signals. The changes of night-time lights and the location-service request show a log-linear positive correlation at both the grid and county levels. (3) The county-level magnitude of the human activities and the changes of night-time lights show significant spatial differentiation across our study area, revealing the diversity of the two signals in response to the typhoon, due to the complicated impact of the typhoon, the complexity of human activity dynamics, various underlying driving forces of anthropogenic NTL signals, as well as inter-regional differences of resistance and resilience ability in response to the typhoon. (4) The damage estimates are positively correlated with the changes of the location-service request and the nighttime lights, indicating that using the two signals as potential proxies to infer hazard-induced damages and losses might be a breakthrough, in particular when other ancillary data are integrated.

Supplementary Materials: The following are available online at http://www.mdpi.com/2072-4292/11/18/2091/s1.

Author Contributions: Conceptualization: Z.L.; data curation: Z.L., Y.D., J.Y, T.M., and T.P.; formal analysis: Z.L., Y.D., F.L., and T.M.; funding acquisition: Y.D. and T.P.; investigation: Z.L., Y.D., and J.Y.; methodology: Z.L., Y.D., F.L., and T.M.; project administration: Y.D., J.Y., and T.P.; resources: Z.L., Y.D., J.Y., and F.L.; software: Z.L.; supervision: Z.L., Y.D., J.Y., F.L., T.M., and T.P.; validation: Z.L., Y.D., J.Y., and F.L.; visualization: Z.L. and F.L.; writing—original draft: Z.L.; writing—review and editing: Z.L., Y.D., J.Y., F.L., and T.M.

Funding: This research was funded by the National Key Research and Development Program (grant number 2017YFB0503605); the National Key R\&D Program of China (grant number 2017YFC1503003); the Key Research Program of Frontier Science, Chinese Academy of Sciences (grant number No.QYZDY-SSW-DQC007); Innovation Program of State Key Laboratory of Resources and Environmental Information System, Chinese Academy of Sciences (grant number 088RA500PA).

Acknowledgments: The authors would like to thank the anonymous reviewers for their constructive comments on an earlier version of this paper.

Conflicts of Interest: The authors declare no conflict of interest.

\section{References}

1. Voigt, S.; Giulio-Tonolo, F.; Lyons, J.; Kučera, J.; Jones, B.; Schneiderhan, T.; Platzeck, G.; Kaku, K.; Hazarika, M.K.; Czaran, L. Global trends in satellite-based emergency mapping. Science 2016, 353, 247-252. [CrossRef]

2. Novellino, A.; Jordan, C.; Ager, G.; Bateson, L.; Fleming, C.; Confuorto, P. Remote Sensing for Natural or Man-Made Disasters and Environmental Changes. In Geological Disaster Monitoring Based on Sensor Networks; Springer Natural Hazards: Singapore, 2019; pp. 23-31. 
3. Mengistu, F.; Suryabhagavan, K.; Raghuvanshi, T.K.; Lewi, E. Landslide Hazard Zonation and Slope Instability Assessment using Optical and InSAR Data: A Case Study from Gidole Town and its Surrounding Areas, Southern Ethiopia. Remote Sens. Land 2019, 3, 1-14. [CrossRef]

4. Klemas, V. Remote sensing of floods and flood-prone areas: An overview. J. Coast. Res. 2014, 31, $1005-1013$. [CrossRef]

5. Dell'Acqua, F.; Gamba, P. Remote sensing and earthquake damage assessment: Experiences, limits, and perspectives. Proc. IEEE 2012, 100, 2876-2890. [CrossRef]

6. Román, M.O.; Wang, Z.; Sun, Q.; Kalb, V.; Miller, S.D.; Molthan, A.; Schultz, L.; Bell, J.; Stokes, E.C.; Pandey, B. NASA's Black Marble nighttime lights product suite. Remote Sens. Environ. 2018, 210, 113-143. [CrossRef]

7. Gillespie, T.W.; Frankenberg, E.; Fung Chum, K.; Thomas, D. Night-time lights time series of tsunami damage, recovery, and economic metrics in Sumatra, Indonesia. Remote Sens. Lett. 2014, 5, 286-294. [CrossRef]

8. Huang, Q.; Yang, X.; Gao, B.; Yang, Y.; Zhao, Y. Application of DMSP/OLS nighttime light images: A meta-analysis and a systematic literature review. Remote Sens. 2014, 6, 6844-6866. [CrossRef]

9. Li, X.; Zhan, C.; Tao, J.; Li, L. Long-term monitoring of the impacts of disaster on human activity using dmsp/ols nighttime light data: A case study of the 2008 Wenchuan, china earthquake. Remote Sens. 2018, 10, 588. [CrossRef]

10. Cao, C.; Shao, X.; Uprety, S. Detecting light outages after severe storms using the S-NPP/VIIRS day/night band radiances. IEEE Geosci. Remote Sens. Lett. 2013, 10, 1582-1586. [CrossRef]

11. Cole, T.; Wanik, D.; Molthan, A.; Román, M.; Griffin, R. Synergistic use of nighttime satellite data, electric utility infrastructure, and ambient population to improve power outage detections in urban areas. Remote Sens. 2017, 9, 286. [CrossRef]

12. Wang, Z.; Román, M.; Sun, Q.; Molthan, A.; Schultz, L.; Kalb, V. Monitoring disaster-related power outages using NASA black marble nighttime light product. ISPRS Int. Arch. Photogramm. Remote Sens. Spat. Inf. Sci 2018, 1853-1856. [CrossRef]

13. Zhao, X.; Yu, B.; Liu, Y.; Yao, S.; Lian, T.; Chen, L.; Yang, C.; Chen, Z.; Wu, J. NPP-VIIRS DNB daily data in natural disaster assessment: Evidence from selected case studies. Remote Sens. 2018, 10, 1526. [CrossRef]

14. Mohamadi, B.; Chen, S.; Liu, J. Evacuation Priority Method in Tsunami Hazard Based on DMSP/OLS Population Mapping in the Pearl River Estuary, China. ISPRS Int. J. Geoinf. 2019, 8, 137. [CrossRef]

15. Li, J.; He, Z.; Plaza, J.; Li, S.; Chen, J.; Wu, H.; Wang, Y.; Liu, Y. Social media: New perspectives to improve remote sensing for emergency response. Proc. IEEE 2017, 105, 1900-1912. [CrossRef]

16. Liu, Y.; Liu, X.; Gao, S.; Gong, L.; Kang, C.; Zhi, Y.; Chi, G.; Shi, L. Social sensing: A new approach to understanding our socioeconomic environments. Ann. Assoc. Am. Geogr. 2015, 105, 512-530. [CrossRef]

17. Kwan, M.-P. Algorithmic geographies: Big data, algorithmic uncertainty, and the production of geographic knowledge. Ann. Am. Assoc. Geogr. 2016, 106, 274-282.

18. Kryvasheyeu, Y.; Chen, H.; Obradovich, N.; Moro, E.; Van Hentenryck, P.; Fowler, J.; Cebrian, M. Rapid assessment of disaster damage using social media activity. Sci. Adv. 2016, 2, e1500779. [CrossRef]

19. Thomas, D.S. The Role of Geographic Information Science \& Technology in Disaster Management. In Handbook of Disaster Research; Springer: Cham, Switzerland, 2018; pp. 311-330.

20. Lampos, V.; Cristianini, N. Nowcasting events from the social web with statistical learning. Acm Trans. Intell. Syst. Technol. (TIST) 2012, 3, 72. [CrossRef]

21. Kropivnitskaya, Y.; Tiampo, K.F.; Qin, J.; Bauer, M.A. Real-time Earthquake Intensity Estimation Using Streaming Data Analysis of Social and Physical Sensors. In Earthquakes and Multi-Hazards around the Pacific Rim; Springer International Publishing: New York, NY, USA, 2018; Volume I, pp. 137-155.

22. Preis, T.; Moat, H.S.; Bishop, S.R.; Treleaven, P.; Stanley, H.E. Quantifying the digital traces of Hurricane Sandy on Flickr. Sci. Rep. 2013, 3, 3141. [CrossRef]

23. Lu, X.S.; Zhou, M.; Qi, L. Analyzing temporal-spatial evolution of rare events by using social media data. In Proceedings of the 2017 IEEE International Conference on Systems, Man and Cybernetics (SMC), Banff, AB, Canada, 5-8 October 2017; pp. 2684-2689.

24. Roy, K.C.; Cebrian, M.; Hasan, S. Quantifying human mobility resilience to extreme events using geo-located social media data. EPJ Data Sci. 2019, 8, 18. [CrossRef]

25. Martín, Y.; Li, Z.; Cutter, S.L. Leveraging Twitter to gauge evacuation compliance: Spatiotemporal analysis of Hurricane Matthew. PLoS ONE 2017, 12, e0181701. [CrossRef] 
26. Hultquist, C.; Simpson, M.; Cervone, G.; Huang, Q. Using nightlight remote sensing imagery and twitter data to study power outages. In Proceedings of the 1st ACM SIGSPATIAL International Workshop on the Use of GIS in Emergency Management, Seattle, WA, USA, 3 November 2015; pp. 1-6.

27. Cervone, G.; Schnebele, E.; Waters, N.; Moccaldi, M.; Sicignano, R. Using Social Media and Satellite Data for Damage Assessment in Urban Areas During Emergencies. In Seeing Cities through Big Data: Research, Methods and Applications in Urban Informatics; Thakuriah, P., Tilahun, N., Zellner, M., Eds.; Springer Geography: Cham, Switzerland, 2017; pp. 443-457.

28. Cervone, G.; Sava, E.; Huang, Q.; Schnebele, E.; Harrison, J.; Waters, N. Using Twitter for tasking remote-sensing data collection and damage assessment: 2013 Boulder flood case study. Int. J. Remote Sens. 2016, 37, 100-124. [CrossRef]

29. Restrepo-Estrada, C.; De Andrade, S.C.; Abe, N.; Fava, M.C.; Mendiondo, E.M.; De Albuquerque, J.P. Geo-social media as a proxy for hydrometeorological data for streamflow estimation and to improve flood monitoring. Comput. Geosci. 2018, 111, 148-158. [CrossRef]

30. Smith, L.; Liang, Q.; James, P.; Lin, W. Assessing the utility of social media as a data source for flood risk management using a real-time modelling framework. J. Flood Risk Manag. 2015, 10, 370-380. [CrossRef]

31. Zhang, N.; Chen, H.; Chen, J.; Chen, X. Social media meets big urban data: A case study of urban waterlogging analysis. Comput. Intell. Neurosci. 2016, 3264587, 1-9. [CrossRef]

32. Rosser, J.F.; Leibovici, D.; Jackson, M. Rapid flood inundation mapping using social media, remote sensing and topographic data. Nat. Hazards 2017, 87, 103-120. [CrossRef]

33. Panteras, G.; Cervone, G. Enhancing the temporal resolution of satellite-based flood extent generation using crowdsourced data for disaster monitoring. Int. J. Remote Sens. 2018, 39, 1459-1474. [CrossRef]

34. McDougall, K.; Temple-Watts, P. The use of LIDAR and volunteered geographic information to map flood extents and inundation. ISPRS Ann. Photogramm. Remote Sens. Spat. Inf. Sci. 2012, 1, 251-256. [CrossRef]

35. Huang, X.; Wang, C.; Li, Z. A near real-time flood-mapping approach by integrating social media and post-event satellite imagery. Ann. GIS 2018, 24, 113-123. [CrossRef]

36. Brouwer, T.; Eilander, D.; Van Loenen, A.; Booij, M.J.; Wijnberg, K.M.; Verkade, J.S.; Wagemaker, J. Probabilistic flood extent estimates from social media flood observations. Nat. Hazards Earth Syst. Sci. 2017, 17, 735. [CrossRef]

37. Sun, D.; Li, S.; Zheng, W.; Croitoru, A.; Stefanidis, A.; Goldberg, M. Mapping floods due to Hurricane Sandy using NPP VIIRS and ATMS data and geotagged Flickr imagery. Int. J. Digit. Earth 2016, 9, 427-441. [CrossRef]

38. Li, Z.; Wang, C.; Emrich, C.T.; Guo, D. A novel approach to leveraging social media for rapid flood mapping: A case study of the 2015 South Carolina floods. Cartogr. Geogr. Inf. Sci. 2018, 45, 97-110. [CrossRef]

39. Yu, Z.; Yao, Y.; Yang, G.; Wang, X.; Vejre, H. Spatiotemporal patterns and characteristics of remotely sensed region heat islands during the rapid urbanization (1995-2015) of Southern China. Sci. Total Environ. 2019, 674, 242-254. [CrossRef]

40. Peng, D.; Hill, E.M.; Li, L.; Switzer, A.D.; Larson, K.M. Application of GNSS interferometric reflectometry for detecting storm surges. GPS Solut. 2019, 23, 47. [CrossRef]

41. National Meteorological Center of China Meteorological Administration. Available online: http://typhoon. nmc.cn/web.html (accessed on 3 November 2018).

42. NOAA Comprehensive Large Array-Data Stewardship System (CLASS). Available online: https://www.bou. class.noaa.gov/saa/products/welcome (accessed on 3 November 2018).

43. NASA EARTHDATA powered by the Earth Observing System Data and Information System (EOSDIS). Available online: https://earthdata.nasa.gov/ (accessed on 3 November 2018).

44. Miller, S.D.; Turner, R.E. A dynamic lunar spectral irradiance data set for NPOESS/VIIRS day/night band nighttime environmental applications. IEEE Trans. Geosci. Remote Sens. 2009, 47, 2316-2329. [CrossRef]

45. Tencent's location-aware data portal. Available online: http://heat.qq.com (accessed on 3 to 31 August 2018).

46. Ma, T. Quantitative responses of satellite-derived night-time light signals to urban depopulation during Chinese New Year. Remote Sens. Lett. 2019, 10, 139-148. [CrossRef]

47. Ma, T. Multi-Level Relationships between Satellite-Derived Nighttime Lighting Signals and Social Media-Derived Human Population Dynamics. Remote Sens. 2018, 10, 1128. [CrossRef]

48. Liu, Z.; Du, Y.; Yi, J.; Liang, F.; Ma, T.; Pei, T. Quantitative estimates of collective geo-tagged human activities in response to typhoon Hato using location-aware big data. Int. J. Digit. Earth 2019, 1-21. [CrossRef] 
49. National Hurricane Center. Available online: https://www.nhc.noaa.gov/gis (accessed on 3 November 2018).

50. DeMaria, M.; Knaff, J.A.; Knabb, R.; Lauer, C.; Sampson, C.R.; DeMaria, R.T. A new method for estimating tropical cyclone wind speed probabilities. Weather Forecast. 2009, 24, 1573-1591. [CrossRef]

51. DeMaria, M.; Knaff, J.A.; Brennan, M.J.; Brown, D.; Knabb, R.D.; DeMaria, R.T.; Schumacher, A.; Lauer, C.A.; Roberts, D.P.; Sampson, C.R. Improvements to the operational tropical cyclone wind speed probability model. Weather Forecast. 2013, 28, 586-602. [CrossRef]

52. National Oceanic and Atmospheric Administration (NOAA). Available online: https://gis.ncdc.noaa.gov/ maps/ncei/cdo/hourly (accessed on 3 November 2018).

53. Yeh, J.-R.; Shieh, J.-S.; Huang, N.E. Complementary ensemble empirical mode decomposition: A novel noise enhanced data analysis method. Adv. Adapt. Data Anal. 2010, 2, 135-156. [CrossRef]

54. Wu, Z.; Huang, N.E. Ensemble empirical mode decomposition: A noise-assisted data analysis method. Adv. Adapt. Data Anal. 2009, 1, 1-41. [CrossRef]

55. Brunsdon, C.; Fotheringham, A.S.; Charlton, M.E. Geographically weighted regression: A method for exploring spatial nonstationarity. Geogr. Anal. 1996, 28, 281-298. [CrossRef]

56. Fast EEMD Package. Available online: https://in.ncu.edu.tw/ \{\}ncu34951/research1.htm (accessed on 3 November 2018).

57. The R Development Core Team. Version 2.6.2; R: A Language and Environment for Statistical Computing; R Foundation for Statistical Computing: Vienna, Austria, 2013.

58. Zeileis, A.; Leisch, F.; Hornik, K.; Kleiber, C. strucchange. An R package for testing for structural change in linear regression models. J. Stat. Softw. 2002, 7, 1-38. [CrossRef]

59. Qi, L.; Li, J.; Wang, Y.; Gao, X. Urban Observation: Integration of Remote Sensing and Social Media Data. IEEE J. Sel. Top. Appl. Earth Obs. Remote Sens. 2019. [CrossRef]

60. Bennett, M.M.; Smith, L.C. Advances in using multitemporal night-time lights satellite imagery to detect, estimate, and monitor socioeconomic dynamics. Remote Sens. Environ. 2017, 192, 176-197. [CrossRef]

(C) 2019 by the authors. Licensee MDPI, Basel, Switzerland. This article is an open access article distributed under the terms and conditions of the Creative Commons Attribution (CC BY) license (http://creativecommons.org/licenses/by/4.0/). 